\title{
The Communication Complexity of Correlation
}

\author{
Prahladh Harsha, Rahul Jain, David McAllester, and Jaikumar Radhakrishnan
}

\begin{abstract}
Let $\mathcal{X}$ and $\mathcal{Y}$ be finite non-empty sets and $(X, Y)$ a pair of random variables taking values in $\mathcal{X} \times \mathcal{Y}$. We consider communication protocols between two parties, Alice and Bob, for generating $X$ and $Y$. Alice is provided an $x \in \mathcal{X}$ generated according to the distribution of $X$, and is required to send a message to Bob in order to enable him to generate $y \in \mathcal{Y}$, whose distribution is the same as that of $\left.Y\right|_{X=x}$. Both parties have access to a shared random string generated in advance. Let $T(X: Y)$ be the minimum (over all protocols) of the expected number of bits Alice needs to transmit to achieve this. We show that
\end{abstract}

$I[X: Y] \leq T[X: Y] \leq I[X: Y]+2 \log _{2}(I[X: Y]+1)+O(1)$.

We also consider the worst-case communication required for this problem, where we seek to minimize the average number of bits Alice must transmit for the worst-case $x \in \mathcal{X}$. We show that the communication required in this case is related to the capacity $\mathcal{C}(E)$ of the channel $E$, derived from $(X, Y)$, that maps $x \in \mathcal{X}$ to the distribution of $\left.Y\right|_{X=x}$. We show that the required communication $T(E)$ satisfies

$$
\mathcal{C}(E) \leq T(E) \leq \mathcal{C}(E)+2 \log _{2}(\mathcal{C}(E)+1)+O(1) .
$$

Using the first result, we derive a direct sum theorem in communication complexity that substantially improves the previous such result shown by Jain, Radhakrishnan and Sen [In Proc. 30th International Colloquium of Automata, Languages and Programming (ICALP), ser. LNCS, vol. 2719. 2003, pp. 300-315].

These results are obtained by employing a rejection sampling procedure that relates the relative entropy between two distributions to the communication complexity of generating one distribution from the other.

Index Terms-mutual information, relative entropy, rejection sampling, communication complexity, direct-sum

\section{INTRODUCTION}

$\mathbf{L}$ ET $\mathcal{X}$ and $\mathcal{Y}$ be finite non-empty sets, and let $(X, Y)$ be a pair of (correlated) random variables taking values in $\mathcal{X} \times$ $\mathcal{Y}$. Consider the following communication problem between two parties, Alice and Bob. Alice is given a random input $x \in \mathcal{X}$, sampled according to the distribution $X$. (We use the same symbol to refer to a random variable and its distribution.) Alice needs to transmit a message $M$ to Bob so that Bob can generate a value $y \in \mathcal{Y}$, that is distributed according to the conditional distribution $\left.Y\right|_{X=x}$ (i.e., the pair $(x, y)$ has joint distribution $(X, Y)$ ). How many bits must Alice send Bob in

A preliminary version of this paper appeared in Proc. 22nd IEEE Conference on Computational Complexity, 2007 [HJMR07].

Prahladh Harsha and David McAllester are with the Toyota Technological Institute, Chicago, USA. Email: \{prahladh, mcallester\}atti-c.org.

Rahul Jain is with the Institute for Quantum Computing and Dept of Computer Science, Univ. of Waterloo, Waterloo CANADA. Email: rjaineiqc.ca. Part of the work was done while the author was at the Univ. of California, Berkeley.

Jaikumar Radhakrishnan is with the Tata Institute of Fundamental Research, Mumbai, INDIA. Email: jaikumaretifr.res.in. Part of the work was done while the author was at the Toyota Technological Institute, Chicago. any protocol that accomplishes this? It follows from the data processing inequality in information theory that the minimum expected number of bits of communication, which we shall call $T(X: Y)$, is at least the mutual information $I[X: Y]$ between $X$ and $Y$, that is,

$$
I[X: Y] \triangleq H[X]+H[Y]-H[X, Y],
$$

where $H[Z]$ denotes the Shannon entropy of the random variable $Z$. (This lower bound follows from the following sequence of inequalities: $T(X: Y) \geq H[M] \geq I[X: M] \geq$ $I[X: Y]$, where the last inequality is the data processing inequality (cf. [CT91, Page 32, Theorem 2.8.1]) applied to the Markov chain $X \rightarrow M \rightarrow Y$.) In this paper, we are interested in deriving an upper bound in terms of $I[X: Y]$ on the expected length of the communication, which can be viewed as a functional characterization of the quantity $I[X: Y]$.

One can also consider a version of this problem which allows error. Formally, let $T_{\lambda}(X: Y)$ denote the minimum expected number of bits Alice needs to send Bob so that the joint distribution generated by the protocol, which we call $(X, \Pi(X))$, is $\lambda$-close in total variation distance ${ }^{1}$ to the joint distribution $(X, Y)$.

This problem was first studied by Wyner [Wyn75], who considered its asymptotic version (with error), where Alice is given several independently drawn samples $\left(x_{1}, \ldots, x_{m}\right)$ from the distribution $X^{m}$ and Bob needs to generate $\left(y_{1}, \ldots, y_{m}\right)$ such that the output distribution of $\left(\left(x_{1}, y_{1}\right), \ldots,\left(x_{m}, y_{m}\right)\right)$ is $\lambda$-close to the distribution $(X, Y)^{m}$. Wyner referred to the amortized minimum expected number of bits Alice needs to send Bob as the common information $C(X: Y)$ of the random variables $X$ and $Y$, i.e.,

$$
C(X: Y) \triangleq \liminf _{\lambda \rightarrow 0}\left[\lim _{m \rightarrow \infty} \frac{T_{\lambda}\left(X^{m}: Y^{m}\right)}{m}\right] .
$$

He then obtained the following remarkable information theoretic characterization of common information.

Theorem I.1 (Wyner's theorem [Wyn75, Theorem 1.3]).

$$
C(X: Y)=\min _{W} I[X Y: W],
$$

where the minimum is taken over all random variables $W$ such that $X$ and $Y$ are conditionally independent given $W$ (in other words, $X \rightarrow W \rightarrow Y$ forms a Markov chain).

It can easily be verified that $T(X: Y) \geq C(X: Y) \geq$ $I[X: Y]$ (See Section VI for a proof of these inequalities). However, as we show in Section Section VI, both these inequalities can be very loose. Thus, this seemingly natural

\footnotetext{
${ }^{1}$ The total variation distance between two distribution $P$ and $Q$ is defined as $\max _{S \subseteq \mathcal{X}}|P(S)-Q(S)|$, which is also equal to $\frac{1}{2}\|P-Q\|_{1}$ where $\|\cdot\|_{1}$ is the $\ell_{1}$-norm.
} 
problem does not offer us the functional characterization for $I[X: Y]$ we were initially hoping for.

\section{A. Protocols with shared randomness}

Our first result shows that there is such a characterization if Alice and Bob are allowed to share random information, generated independently of Alice's input. In fact, then Alice need send no more than approximately $I[X: Y]$ bits to Bob. In order to state our result precisely, let us first define the kind of communication protocol Alice and Bob are expected to use.

Definition I.2 (One-way protocol). In a one-way protocol, the two parties Alice and Bob share a random string $R$, and also have private random strings $R_{A}$ and $R_{B}$ respectively. Alice receives an input $x \in \mathcal{X}$. Based on the shared random string $R$ and her own private random string $R_{A}$, she sends a message $M\left(x, R, R_{A}\right)$ to Bob. On receiving the message $M$, Bob computes the output $y=y\left(M, R, R_{B}\right)$. The protocol is thus specified by the two functions $M\left(x, R, R_{A}\right)$ and $y\left(M, R, R_{B}\right)$ and the distributions for the random strings $R, R_{A}$ and $R_{B}$. For such a protocol $\Pi$, let $\Pi(x)$ denote its (random) output when the input given to Alice is $x$. Let $T_{\Pi}(x)$ be the expected length of the message transmitted by Alice to Bob, that is, $T_{\Pi}(x)=\mathbb{E}\left[\left|M\left(x, R, R_{A}\right)\right|\right]$. Note that the private random strings can be considered part of the shared random string if we are not concerned about minimizing the amount of shared randomness.

One can also consider protocols with multiple rounds of communication. However, if our goal is only to minimize communication, then one can assume without loss of generality that the protocol is one-way. This is because we can include the random strings $R_{A}$ and $R_{B}$ as part of the shared random string $R$, enabling Alice to determine Bob's responses to her messages on her own. She can then concatenate all her messages and send them in one round.

Definition I.3. Given random variables $(X, Y)$, let

$$
T_{\lambda}^{R}(X: Y) \triangleq \min _{\Pi} \underset{x \leftarrow X}{\mathbb{E}}\left[T_{\Pi}(x)\right],
$$

where $\Pi$ ranges over all one-way protocols where $(X, \Pi(X))$ is $\lambda$-close in total variation distance to the distribution $(X, Y)$. For the special case when $\lambda=0$, we write $T^{R}(X: Y)$ instead of $T_{0}^{R}(X: Y)$.

As in the case of $T(X: Y)$, the data processing inequality implies that $T^{R}(X: Y)$ is at least $I(X: Y)$; indeed, we have $T^{R}(X: Y) \geq H[M] \geq H[M \mid R] \geq I[X: M \mid R]=I[X:$ $M \mid R]+I[X: R]=I[X: M R] \geq I[X: Y]$, where we use the fact that $X$ and $R$ are independent to conclude that $I[X$ : $R]=0$, the chain rule for mutual information and the data processing inequality (this time applied to the Markov chain $X \rightarrow(M, R) \rightarrow Y)$ to conclude that $I[X: M R] \geq I[X: Y]$.

Our first result shows that this lower bound is essentially tight, giving a characterization of mutual information (modulo some lower order logarithmic terms ${ }^{2}$ ).

\footnotetext{
${ }^{2}$ All logarithms (denoted by $\mathrm{lg}$ ) in this paper are with respect to base 2 .
}

Result 1 (Characterization of mutual information).

$I[X: Y] \leq T^{R}(X: Y) \leq I[X: Y]+2 \lg (I[X: Y]+1)+O(1)$.

We have an additive $2 \lg (I[X: Y]+1)$ term in the upper bound because our proof of the result employs a prefix-free encoding of integers that requires $\lg n+2 \lg \lg (n+1)+O(1)$ bits to encode the positive integer $n$. By using an encoding that requires $\lg n+(1+\varepsilon) \lg \lg (n+1)+O(1)$ bits, the constant 2 can be improved to $(1+\varepsilon)$ for any $\varepsilon>0$.

The above result does not place any bound on the amount of randomness that Alice and Bob need to share. In fact, there exist distributions $(X, Y)$ for which our proof of Result 1 requires Alice and Bob to share a random string of unbounded length. However, by stating the question in terms of flows in a suitably defined network, we can bound the amount of shared randomness by $O(\lg \lg |\mathcal{X}|+\lg |\mathcal{Y}|)$ provided we allow the expected communication to increase by $O(\lg \lg (|\mathcal{Y}|)$ ) (see Section VII).

\section{B. Generating one distribution from another}

The main tool in our proof of Result 1 is a sampling procedure for generating one distribution from another. This sampling procedure is of independent interest because it relates the relative entropy between two distributions and the communication complexity of generating one distribution from the other.

Definition I.4 (relative entropy). The relative entropy or Kullback-Leibler divergence between two probability distributions $P$ and $Q$ on a finite set $\mathcal{X}$ is

$$
S(P \| Q)=\sum_{x \in \mathcal{X}} P(x) \lg \frac{P(x)}{Q(x)} .
$$

Observe that $S(P \| Q)$ is finite if and only if the support of distribution $P$ (i.e., the set of points $x \in \mathcal{X}$ such that $P(x)>$ 0 ) is contained in the support of the distribution $Q$; also, it is zero iff $P=Q$, but is otherwise always positive.

Let $P$ and $Q$ be two distributions such that the relative entropy $S(P \| Q)$ is finite. We consider the problem of generating a sample according to $P$ from a sequence of samples drawn according to $Q$. Let $\left\langle x_{1}, x_{2}, \ldots, x_{i}, \ldots\right\rangle$ be a sequence of samples, drawn independently, each with distribution $Q$. The idea is to generate an index $i^{*}$ (a random variable depending on the sample) so that the sample $x_{i^{*}}$ has distribution $P$. For example, if $P$ and $Q$ are identical, then we can set $i^{*}=1$ and be done. It is easy to show ${ }^{3}$ that for any such procedure

$$
\mathbb{E}\left[\ell\left(i^{*}\right)\right] \geq S(P \| Q),
$$

where $\ell\left(i^{*}\right)$ is the length of the binary encoding of $i^{*}$. We show that there, in fact, exists a procedure that almost achieves this lower bound.

$$
\begin{aligned}
& { }^{3} \text { For all } x \text { and } i, \alpha_{i} \triangleq \operatorname{Pr}\left[i^{*}=i \wedge x_{i}^{*}=x\right] \leq \operatorname{Pr}\left[x_{i}=x\right]=Q(x) \text {; } \\
& \text { thus, } \mathbb{E}\left[\ell\left(i^{*}\right) \mid x_{i^{*}}=x\right] \geq H\left[i^{*} \mid x_{i^{*}}=x\right] \\
& =\sum_{i} \frac{\alpha_{i}}{P(x)} \lg \frac{P(x)}{\alpha_{i}} \geq \lg \frac{P(x)}{Q(x)},
\end{aligned}
$$

and $\mathbb{E}\left[\ell\left(i^{*}\right)\right]=\sum_{x} P(x) \mathbb{E}\left[\ell\left(i^{*}\right) \mid x_{i^{*}}=x\right] \geq S(P \| Q)$. 
Lemma I.5 (Rejection sampling lemma). Let $P$ and $Q$ be two distributions such that $S(P \| Q)$ is finite. There exists a sampling procedure REJ-SAMPLER which on input a sequence $\left\langle x_{1}, x_{2}, \ldots, x_{i}, \ldots\right\rangle$ of independently drawn samples from the distribution $Q$ outputs (with probability 1) an index $i^{*}$ such that the sample $x_{i^{*}}$ is distributed according to the distribution $P$ and the expected encoding length of the index $i^{*}$ is at most

$$
S(P \| Q)+2 \lg (S(P \| Q)+1)+O(1),
$$

where the expectation is taken over the sample sequence and the internal random coins of the procedure REJ-SAMPLER.

As in the case of Result 1, the constant 2 can be improved to any constant $(1+\varepsilon)$ for any $\varepsilon>0$.

\section{Reverse Shannon theorem}

In Result 1, we considered the communication cost averaged over $x \in \mathcal{X}$, chosen according to the distribution of $X$. We now consider the worst-case communication over all $x \in \mathcal{X}$ (but we still average over the random choices of the protocol). Let $\mathcal{X}$ and $\mathcal{Y}$ be finite non-empty sets as before. Let $\mathcal{P}_{\mathcal{Y}}$ be the set of all probability distributions on the set $\mathcal{Y}$. A channel with input alphabet $\mathcal{X}$ and output alphabet $\mathcal{Y}$ is a function $E: \mathcal{X} \rightarrow \mathcal{P}_{\mathcal{Y}}$. The Shannon capacity of such a channel is

$$
\mathcal{C}(E) \triangleq \max _{(X, Y)} I[X: Y]
$$

where $(X, Y)$ is a pair of random variables taking values in $\mathcal{X} \times \mathcal{Y}$ such that for all $x \in \mathcal{X}$ and $y \in \mathcal{Y}, \operatorname{Pr}[Y=y \mid$ $X=x]=E(x)(y)$. A simulator for this channel (using a noiseless communication channel and shared randomness) is a one-way protocol $\Pi$ such that for all $x \in \mathcal{X}$, Bob's output $\Pi(x)$ has distribution $E(x)$. The goal is to minimize the worstcase communication; let

$$
T(E)=\min _{\Pi} \max _{x \in \mathcal{X}} T_{\Pi}(x),
$$

where the minimum is taken over all valid simulators $\Pi$ of $E$. It is easy to show and well known that $T(E) \geq \mathcal{C}(E)$. We show that this lower bound is essentially tight (modulo some lower order logarithmic terms).

Result 2 (One-shot reverse Shannon theorem). $T(E) \leq$ $\mathcal{C}(E)+2 \lg (\mathcal{C}(E)+1)+O(1)$.

Such a result is called the Reverse Shannon Theorem as it gives an (optimal) simulation of noisy channels using noiseless channels and shared randomness. We use the term one-shot to distinguish this result from the previously known asymptotic versions. See Section I-E for a discussion of these results. As in the case of Result 1, the constant 2 can be improved to $(1+\varepsilon)$ for any $\varepsilon>0$.

\section{A direct-sum result in communication complexity}

Result 1 has an interesting consequence in communication complexity. To state this result, we need some standard definitions from two-party communication complexity. We refer the reader to the book by Kushilevitz and Nisan [KN97] for an excellent introduction to communication complexity. Let $\mathcal{X}$,
$\mathcal{Y}$ and $\mathcal{Z}$ be finite non-empty sets, and let $f: \mathcal{X} \times \mathcal{Y} \rightarrow \mathcal{Z}$ be a function. A two-party protocol for computing $f$ consists of two parties, Alice and Bob, who get inputs $x \in \mathcal{X}$ and $y \in \mathcal{Y}$ respectively, and exchange messages in order to compute $f(x, y) \in \mathcal{Z}$. A protocol is said to be $k$-round, if the two parties exchange at most $k$ messages.

For a distribution $\mu$ on $\mathcal{X} \times \mathcal{Y}$, let the $\varepsilon$-error $k$-round distributional communication complexity of $f$ under $\mu$ (denoted by $D_{\varepsilon}^{\mu, k}(f)$ ), be the number of bits communicated (for the worst-case input) by the best deterministic $k$-round protocol for $f$ with average error at most $\varepsilon$ under $\mu$. Let $R_{\varepsilon}^{\text {pub }, k}(f)$, the public-coins $k$-round randomized communication complexity of $f$ with worst case error $\varepsilon$, be the number of bits communicated (for the worst-case input) by the best $k$-round publiccoins randomized protocol, that for each input $(x, y)$ computes $f(x, y)$ correctly with probability at least $1-\varepsilon$. Randomized and distributional complexity are related by the following celebrated result of Yao [Yao77].

Theorem I.6 (Yao's minmax principle [Yao77]). $R_{\varepsilon}^{\text {pub, } k}(f)=$ $\max _{\mu} D_{\varepsilon}^{\mu, k}(f)$

For function $f: \mathcal{X} \times \mathcal{Y} \rightarrow \mathcal{Z}$, the $t$-fold direct sum of $f$, $f^{(t)}: \mathcal{X}^{t} \times \mathcal{Y}^{t} \rightarrow \mathcal{Z}^{t}$, is defined by

$$
f^{(t)}\left(\left\langle x_{1}, \ldots, x_{t}\right\rangle,\left\langle y_{1}, \ldots, y_{t}\right\rangle\right) \triangleq\left\langle f\left(x_{1}, y_{1}\right), \ldots, f\left(x_{t}, y_{t}\right)\right\rangle .
$$

It is natural to ask if the communication complexity of $f^{(t)}$ is at least $t$ times that of $f$. This is commonly known as the direct sum question. The direct sum question is a very basic question in communication complexity and had been studied for a long time. Several results are known for this question in restricted settings for deterministic and randomized protocols [KN97]. Recently Chakrabarti, Shi, Wirth and Yao [CSWY01] studied this question in the simultaneous message passing (SMP) model in which Alice and Bob, instead of communicating with each other, send a message each to a third party Referee who then outputs a $z$ such that $f(x, y)=z$. They showed that in this model, the Equality function $\mathrm{EQ}$ satisfies the direct sum property. Their result also holds for any function that satisfies a certain robustness requirement. Jain, Radhakrishnan and Sen [JRS05] showed that the claim holds for all functions and relations, not necessarily those satisfying the robustness condition, both in the one-way and the SMP model of communication. In another work Jain, Radhakrishnan and Sen [JRS03a] showed a weaker direct sum result for boundedround two-way protocols under product distributions over the inputs. Their result was the following (here $\mu$ is a product distribution on $\mathcal{X} \times \mathcal{Y}$ and $k$ represents the number of rounds):

$$
D_{\varepsilon}^{\mu^{t}, k}\left(f^{(t)}\right) \geq t\left(\frac{\delta^{2}}{2 k} \cdot D_{\varepsilon+2 \delta}^{\mu, k}(f)-2\right)
$$

We show that Result 1 implies the following stronger claim.

Result 3 (Direct sum for communication complexity). For any function $f: \mathcal{X} \times \mathcal{Y} \rightarrow \mathcal{Z}$, and a product distribution $\mu$ on $\mathcal{X} \times \mathcal{Y}$, we have

$$
D_{\varepsilon}^{\mu^{t}, k}\left(f^{(t)}\right) \geq \frac{t}{2}\left(\delta D_{\varepsilon+\delta}^{\mu, k}(f)-O(k)\right) .
$$


Applying Yao's minimax principle (Theorem I.6), we have:

$$
R_{\varepsilon}^{\mathrm{pub}, k}\left(f^{(t)}\right) \geq \max _{\mu}\left(\frac{t}{2}\left(\delta D_{\varepsilon+\delta}^{\mu, k}(f)-O(k)\right)\right) .
$$

where the maximum above is taken over all product distributions $\mu$ on $\mathcal{X} \times \mathcal{Y}$.

Result 3 requires the distribution $\mu$ to be a product distribution. If this requirement could be removed, we would be able infer a direct sum result for randomized communication complexity, namely

$$
R_{\varepsilon}^{\mathrm{pub}, k}\left(f^{(t)}\right) \geq \frac{t}{2}\left(\delta R_{\varepsilon+\delta}^{\mathrm{pub}, k}(f)-O(k)\right) .
$$

In some cases, however, our result implies this claim: if for some function $f$, the distribution $\mu$ that achieves the maximum in Theorem I.6 when applied to $R_{\varepsilon+\delta}^{\mathrm{pub}, k}(f)$ is a product distribution, then (I.2) holds.

\section{E. Related work}

Asymptotic versions of our Results 1 and 2 were shown (independently of our work) by Winter [Win02] and Bennett $e t$ al. [BSST02] respectively.

Theorem I.7 ([Win02, Theorem 9 and Remark 10]). For every pair of distributions $(X, Y)$ and $\lambda>0$ and n, there exists a one-way protocol $\Pi_{n}$ such that the distribution $\left(X^{n}, \Pi_{n}\left(X^{n}\right)\right)$ is $\lambda$-close in total variation distance to the joint distribution $\left(X^{n}, Y^{n}\right)$ and furthermore,

$$
\max _{\bar{x} \in \mathcal{X}^{n}} T_{\Pi_{n}}(\bar{x}) \leq n I[X: Y]+O\left(\frac{1}{\lambda}\right) \cdot \sqrt{n} .
$$

Theorem I.8 (Reverse Shannon theorem [BSST02]). Let $E$ be a discrete memoryless channel with Shannon capacity $C$ and $\varepsilon>0$. Then, for each block size $n$ there is a deterministic simulation protocol $\Pi_{n}$ for $E^{n}$ which makes use of a noiseless channel and prior random information $R$ shared between sender and receiver. The simulation is exactly faithful in the sense that for all $n$, and for all $\bar{x} \in \mathcal{X}^{n}$, the output $\Pi_{n}(\bar{x})$ has the distribution $E^{n}(\bar{x})$, and it is asymptotically efficient in the sense that

$$
\lim _{n \rightarrow \infty} \max _{\bar{x} \in \mathcal{X}^{n}} \operatorname{Pr}\left[T_{\Pi_{n}}(\bar{x})>n(C(E)+\varepsilon)\right]=0 .
$$

Note that the asymptotic result of Winter [Win02] is slightly stronger than what is stated above in that it actually bounds the worst case number of bits communicated while our results (and the above statement) bound the expected number of bits communicated. Despite this, these asymptotic results (and their stronger counterparts) follow immediately from our results by routine applications of the law of large numbers.

a) One-shot vs. asymptotic results: In the light of the above, it might seem natural to ask why one should be interested in one-shot versions of known asymptotic results. Our motivation for the one-shot versions is two-fold.

- The asymptotic equipartition property (cf. [CT91, Chapter 3]) for distributions states that for sufficiently large $n, n$ independently drawn samples from a distribution $X$ almost always fall in what are called "typical sets".
Typical sets have the property that all elements in it are nearly equiprobable and the size of the typical set is approximately $2^{n H[X]}$. Any property that is proved for typical sets will then be true with high probability for a large sequence of independently drawn samples. Thus, to prove the asymptotic results, it suffices to prove the same for typical sets. Thus, one might contend that these asymptotic results are in fact properties of typical sets and it could be the case that the results are in fact, not true for the one-shot case. Our results show that this is not the case and one need not resort to typical sets to prove them.

- Second, our results provide tools for certain problems in communication complexity (e.g., our improved direct sum result). For such communication complexity applications, the asymptotic versions do not seem to suffice and we require the one-shot versions.

b) Bounding shared randomness: As mentioned earlier, we can bound the shared randomness in Result 1 by $O(\lg |\mathcal{X}|+\lg |\mathcal{Y}|)$ if we are allowed to increase the expected communication by an additive term of $O(\lg \lg (|\mathcal{X}|+\mathcal{Y} \mid))$ (see Section VII). This raises the natural question of tradeoffs between shared randomness and expected communication. The asymptotic version of this problem was recently solved by Bennett and Winter (Personal Communication [BW06]).

c) Substate Theorem: Jain, Radhakrishnan and Sen [JRS02] prove the following result relating the relative entropy between two distributions $P$ and $Q$ to how well a distribution is contained in another.

Theorem I.9 (Classical substate theorem, [JRS02]). Let $P$ and $Q$ be two distributions such that $k=S(P \| Q)$ is finite. For all $\varepsilon>0$ there exists a distribution $P^{\prime}$ such that $\left\|P^{\prime}-P\right\|_{1} \leq \varepsilon$ and $Q=\alpha P^{\prime}+(1-\alpha) P^{\prime \prime}$ where $P^{\prime \prime}$ is some other distribution and $\alpha=2^{-O(k / \varepsilon)}$.

The rejection sampling lemma (Lemma I.5) is a strengthening of the above theorem (the above theorem follows from Lemma I.5 by an application of Markov's inequality). In fact, the classical substate theorem can then be used to prove a weaker version of Result 1 and Result 2 which allows for error. More precisely, one can infer (from Theorem I.9) that $\left.T_{\lambda}^{R}(X: Y) \leq O(I[X: Y]) / \lambda\right)$ and $T_{\lambda}^{R}(E) \leq O(\mathcal{C}(E) / \lambda)$. Note Jain, Radhakrishnan and Sen [JRS02] actually showed a quantum analogue of the above substate theorem. It is open if there exist quantum analogues of our results.

d) Lower Bounds using message compression: Chakrabarti and Regev [CR04] prove that any randomized cell probe algorithm that solves the approximate nearest search problem on the Hamming cube $\{0,1\}^{d}$ using polynomial storage and word size $d^{O(1)}$ requires a worst case query time of $\Omega(\lg \lg d / \lg \lg \lg d)$. An important component in their proof of this lower bound is the message compression technique of Jain, Radhakrishnan and Sen [JRS03a]. The rejection sampling lemma (Lemma I.5) can be used to improve message compression of [JRS03a], which in turn simplifies the lower bound argument of Chakrabarti and Regev. It is likely that there are other similar applications. 


\section{Organization}

The rest of the paper is organized as follows: We first prove Results 1 and 2 assuming the rejection sampling lemma (Lemma I.5) in Sections II and III respectively. We then proceed to prove the rejection sampling lemma in Section IV. The Direct Sum Result (Result 3) is then proved in Section V. In Section VI, we give examples of joint distributions $(X, Y)$ that satisfy $T(X: Y)=\omega(C(X: Y))$ and $C(X: Y)=$ $\omega(I[X: Y])$. Finally, in Section VII, we show how to reduce the shared randomness at the expense of a small additive cost in the expected communication.

\section{PROOF OF Result 1}

Result 1 follows easily from the rejection sampling lemma (Lemma I.5) and the following well-known relationship between relative entropy and mutual information.

Fact II.1. $I[X: Y]=\mathbb{E}_{x \leftarrow X}\left[S\left(\left.Y\right|_{X=x} \| Y\right)\right]$.

In other words, the mutual information between any two random variables $X$ and $Y$ is the average relative entropy between the conditional distribution $\left.Y\right|_{X=x}$ and the marginal distribution $Y$.

Proof of Result 1: We may assume that the random string shared by Alice and Bob is a sequence of independently drawn samples $\left\langle y_{1}, y_{2}, \ldots\right\rangle$ according to the marginal distribution $Y$. On input $x \in \mathcal{X}$ drawn according to the distribution $X$, Alice uses the sampling procedure REJSAMPLER (from Lemma I.5) to sample the conditional distribution $\left.Y\right|_{X=x}$ from the marginal distribution $Y$ in order to generate the index $i^{*}$. (Note that the conditional and marginal distribution always satisfy $\left.S\left(\left.Y\right|_{X=x} \| Y\right)<\infty\right)$. Alice transmits the index $i^{*}$ to Bob, who then outputs the sample $y_{i^{*}}$ which has the required distribution. The expected number of bits transmitted in this protocol is at most $E_{x \leftarrow X}\left[S\left(\left.Y\right|_{X=x} \| Y\right)+2 \lg \left(S\left(\left.Y\right|_{X=x} \| Y\right)+1\right)+O(1)\right]$ which (by Fact II.1 and Jensen's inequality) is at most $I[X: Y]+2 \lg (I[X: Y]+1)+O(1)$.

\section{PROOF OF THE ONE-SHOT REVERSE SHANNON THEOREM (RESULT 2)}

Fix the channel $E$, and let $(X, Y)$ be the random variables that realize its channel capacity. Let $Q$ be the marginal distribution of $Y$.

Claim III.1. For all $x \in \mathcal{X}, S(E(x) \| Q) \leq \mathcal{C}(E)$.

The existence of a distribution $Q$ with the above property was also shown by Jain [Jai06] using a different argument.

Note that the result follows immediately from this claim by invoking the rejection sampling lemma (Lemma I.5). The resulting protocol uses samples drawn according to $Q$ as shared randomness and on input $x \in \mathcal{X}$ generates a symbol in $\mathcal{Y}$ whose distribution is $E(x)$. The communication required is bounded by $S(E(x) \| Q)+2 \lg (S(E(x) \| Q)+1)+O(1)$; by Claim III.1, this is at most $\lg \mathcal{C}(E)+2 \lg (\mathcal{C}(E)+1)+O(1)$.

Proof of Claim III.1: For contradiction assume that for some $x_{0} \in \mathcal{X}$, we have $S\left(E\left(x_{0}\right) \| Q\right)>\mathcal{C}(E)$. We will show that by assigning greater probability to $x_{0}$ than it receives in $X$, we can obtain a pair of random variables $\left(X^{\prime}, Y^{\prime}\right)$ whose distribution is compatible with the channel, but whose mutual information is strictly more than $\mathcal{C}(E)-$ a contradiction. Formally, for $\alpha \in[0,1]$, consider the random variable $X_{\alpha}$ obtained by picking $x_{0}$ with probability $\alpha$ and $X$ with probability $(1-\alpha)$. Let $Y_{\alpha}$ be a random variable correlated with $X_{\alpha}$ such that for all $x \in \mathcal{X}$ and $y \in \mathcal{Y}$, $\operatorname{Pr}\left[Y_{\alpha}=y \mid X_{\alpha}=x\right]=E(x)(y)$; let $Q_{\alpha}$ be the marginal distribution of $Y_{\alpha}$. We will show that

$$
\left.\frac{\mathrm{d} I\left[X_{\alpha}: Y_{\alpha}\right]}{\mathrm{d} \alpha}\right|_{\alpha=0}=S\left(E\left(x_{0}\right) \| Q\right)-I[X: Y]>0 .
$$

It then follows that for some small enough $\alpha>0$,

$$
I\left[X_{\alpha}: Y_{\alpha}\right]>I\left[X_{0}: Y_{0}\right]=\mathcal{C}(E),
$$

contradicting our assumption that

$$
I\left[X_{0}: Y_{0}\right]=I[X: Y]=\mathcal{C}(E) .
$$

It remains to establish (III.1).

$$
\begin{aligned}
& I\left[X_{\alpha}: Y_{\alpha}\right] \\
= & \underset{x \leftarrow X_{\alpha}}{\mathbb{E}}\left[S\left(E(x) \| Q_{\alpha}\right)\right] \\
= & \underset{\mathbb{E}}{\mathbb{E}}[S(E(x) \| Q)]-S\left(Q_{\alpha} \| Q\right) \\
= & \alpha S\left(E\left(x_{0}\right) \| Q\right)+(1-\alpha) \underset{x \leftarrow X}{\mathbb{E}}[S(E(x) \| Q)]-S\left(Q_{\alpha} \| Q\right) \\
= & \alpha S\left(E\left(x_{0}\right) \| Q\right)+(1-\alpha) I[X: Y]-S\left(Q_{\alpha} \| Q\right) .
\end{aligned}
$$

Since $Q_{\alpha}(y)=\alpha E\left(x_{0}\right)(y)+(1-\alpha) Q(y)$, we have

$$
\begin{aligned}
S\left(Q_{\alpha} \| Q\right)=\sum_{y \in \mathcal{Y}}\left(\alpha E\left(x_{0}\right)(y)+(1-\alpha) Q(y)\right) . \\
\lg \left(\frac{\alpha E\left(x_{0}\right)(y)+(1-\alpha) Q(y)}{Q(y)}\right) .
\end{aligned}
$$

A quick calculation then yields:

$$
\begin{aligned}
& \frac{\mathrm{d} I\left[X_{\alpha}: Y_{\alpha}\right]}{\mathrm{d} \alpha}=S\left(E\left(x_{0}\right) \| Q\right)-I[X: Y]- \\
& \sum_{y \in \mathcal{Y}}\left(E\left(x_{0}\right)(y)-Q(y)\right) \cdot \lg \left(\frac{\alpha E\left(x_{0}\right)(y)+(1-\alpha) Q(y)}{Q(y)}\right) .
\end{aligned}
$$

Our claim (III.1) follows immediately.

This completes the proof of Result 2.

\section{THE REJECTION SAMPLING PROCEDURE}

Let $P$ and $Q$ be two distributions on the set $\mathcal{X}$ such that the relative entropy $S(P \| Q)$ is finite. Recall that we need to design a rejection sampling procedure that on input a sequence of samples $\left\langle x_{1}, x_{2}, \ldots\right\rangle$ independently drawn according to the distribution $Q$, outputs an index $i^{*}$ such that $x_{i^{*}}$ is distributed according to $P$, and the expected encoding length of the index $i^{*}$ is as small as possible.

The procedure REJ-SAMPLER we formally state below examines the samples $\left\langle x_{i}: i \in \mathbb{N}\right\rangle$ sequentially; after examining $x_{i}$ it either accepts it (by returning the value $i$ for $i^{*}$ ) or moves on to the next sample $x_{i+1}$. For $x \in \mathcal{X}$ and $i \geq 1$, let $\alpha_{i}(x)$ 
denote the probability that the procedure outputs $i$ and $x_{i}=x$. We wish to ensure that for all $x \in \mathcal{X}$,

$$
P(x)=\sum_{i=1}^{\infty} \alpha_{i}(x)
$$

Let $p_{i}(x)=\sum_{j=1}^{i} \alpha_{i}(x)$; thus, $p_{i}(x)$ is the probability that the procedure halts with $i^{*} \leq i$ and $x_{i^{*}}=x$. Let $p_{i}^{*}=\sum_{x \in \mathcal{X}} p_{i}(x)$; hence, $p_{i}^{*}$ is the probability that the procedure halts within $i$ iterations. These quantities will be determined once $\alpha_{i}(x)$ is defined. We define $\alpha_{i}(x)$ (and hence $p_{i}(x)$ and $\left.p_{i}^{*}\right)$ inductively. For $x \in \mathcal{X}$, let $p_{0}(x)=0$. For $i=1,2, \ldots$ and for $x \in \mathcal{X}$, let

$$
\begin{aligned}
\alpha_{i}(x) & =\min \left\{P(x)-p_{i-1}(x),\left(1-p_{i-1}^{*}\right) Q(x)\right\} \\
p_{i}(x) & =p_{i-1}(x)+\alpha_{i}(x) .
\end{aligned}
$$

The definition of $\alpha_{i}(x)$ can be understood as follows. The first term $P(x)-p_{i-1}(x)$ ensures that $p_{i}(x)$ never exceeds $P(x)$. The second term $\left(1-p_{i-1}^{*}\right) Q(x)$ has the following interpretation. The probability that the procedure enters the $i$ th iteration and gets to examine $x_{i}$ is precisely $1-p_{i-1}^{*}$. Since, $\operatorname{Pr}\left[x_{i}=x\right]=Q(x)$, the probability that the procedure outputs $i$ after examining $x_{i}=x$ can be at most $\left(1-p_{i-1}^{*}\right) Q(x)$. Our definition of $\alpha_{i}(x)$ corresponds to the greedy strategy, that accepts the $i$-th sample, with as much probability as possible under the constraint that $p_{i}(x) \leq P(x)$ for all $x \in \mathcal{X}$. The following procedure implements this idea formally.

$\operatorname{REJ}-\operatorname{SAMPLER}(P, Q)$

RANDOM INPUT: $\left\langle x_{i}: i \in \mathbb{N}\right\rangle$ a sequence of samples independently drawn from the distribution $Q$.

A. Compute the sequence $\left\langle p_{i}: i \in \mathbb{N}\right\rangle$ as defined above.

B. For $i \leftarrow 1$ to $\infty$ do

\section{ITERATION $(i)$}

a) Examine sample $x_{i}$.

$\begin{array}{llr}\text { b) With probability } & \beta_{i}\left(x_{i}\right) & = \\ \min \left\{\frac{P\left(x_{i}\right)-p_{i-1}\left(x_{i}\right)}{\left(1-p_{i-1}^{*}\right) Q\left(x_{i}\right)}, 1\right\}, & \text { output } i & \text { and }\end{array}$ halt.

Note that if we arrive at the $i$-th iteration, then $p_{i-1}^{*}<1$; also $Q\left(x_{i}\right)>0$. So, $\beta_{i}$ is well defined.

Note that the probability that this procedure outputs $i$ and $x_{i}=x$ is precisely $\beta_{i}(x)\left(1-p_{i-1}^{*}\right) Q(x)=\alpha_{i}(x)$. We have two claims.

Claim IV.1. For all $x,\left\langle p_{i}(x): i=1,2, \ldots\right\rangle$ converges to $P(x)$.

Claim IV.2. Let $\ell$ be a prefix-free encoding of positive integers such that $\ell(n) \leq \lg n+2 \lg \lg (n+1)+O(1)$. Then, $\mathbb{E}\left[\ell\left(i^{*}\right)\right]=$ $S(P \| Q)+2 \lg (S(P \| Q)+1)+O(1)$.

Proof of Claim IV.1: It is enough to show that for all $x \in \mathcal{X}, p_{i}(x)$ converges to $P(x)$. Fix an $x \in \mathcal{X}$. We will show that for $i=1,2, \ldots$,

$$
\alpha_{i}(x) \geq\left(P(x)-p_{i-1}(x)\right) Q(x)
$$

and then induction to show $P(x)-p_{i}(x) \leq P(x)(1-Q(x))^{i}$ : the inequality holds for $i=0$ because $p_{0}(x)=0$, and for the induction step we have

$$
\begin{aligned}
P(x)-p_{i}(x) & =P(x)-p_{i-1}(x)-\alpha_{i}(x) \\
& \leq\left(P(x)-p_{i-1}(x)\right)(1-Q(x)) \\
& \leq P(x)(1-Q(x))^{i} .
\end{aligned}
$$

Since $S(P \| Q)<\infty$, we have that if $P(x)>0$, then $Q(x)>$ 0 . Since, $P(x) \geq p_{i}(x)$, we conclude that $p_{i}(x)$ converges to $P(x)$. It remains to establish (IV.1). First, observe that

$$
\begin{aligned}
1-p_{i-1}^{*} & =\sum_{y \in \mathcal{X}} P(y)-\sum_{y \in \mathcal{X}} p_{i-1}(y) \\
& =\sum_{y \in \mathcal{X}}\left(P(y)-p_{i-1}(y)\right) \\
& \geq P(x)-p_{i-1}(x) .
\end{aligned}
$$

Now, (IV.1) follows immediately from the definition, $\alpha_{i}(x)=$ $\min \left\{P(x)-p_{i-1}(x),\left(1-p_{i-1}^{*}\right) Q(x)\right\}$.

Proof of Claim IV.2: We show below that

$$
\mathbb{E}\left[\lg i^{*}\right] \leq S(P \| Q)+O(1) .
$$

Then, we have

$$
\begin{aligned}
\mathbb{E}\left[\ell\left(i^{*}\right)\right] & =\mathbb{E}\left[\lg i^{*}+2 \lg \lg \left(i^{*}+1\right)+O(1)\right] \\
& =\mathbb{E}\left[\lg i^{*}\right]+2 \mathbb{E}\left[\lg \lg \left(i^{*}+1\right)\right]+O(1) \\
& \leq \mathbb{E}[\lg i]+2 \lg \left(\mathbb{E}\left[\lg \left(i^{*}+1\right)\right]\right)+O(1)
\end{aligned}
$$

[Jensen's inequality: $\lg (\cdot)$ is concave]

$\leq \mathbb{E}\left[\lg i^{*}\right]+2 \lg \left(\mathbb{E}\left[\lg i^{*}\right]+1\right)+O(1)$

$\leq S(P \| Q)+O(1)+2 \lg (S(P \| Q)+O(1))+O(1)$

[by (IV.2)]

$=S(P \| Q)+2 \lg (S(P \| Q)+1)+O(1)$.

It remains to show (IV.2). We have

$$
\mathbb{E}[\lg i]=\sum_{x \in \mathcal{X}} \sum_{i=1}^{\infty} \alpha_{i}(x) \cdot \lg i
$$

The term corresponding to $i=1$ is 0 . To bound the other terms we need to obtain a suitable bound on $\alpha_{i}(x)$. Let $i \geq 2$ and suppose $\alpha_{i}(x)>0$. Then for $j=1,2, \ldots, i, p_{j-1}(x)<$ $P(x)$, implying that for $j=1,2, \ldots, i-1, \alpha_{j}(x)=(1-$ $\left.p_{j-1}^{*}\right) Q(x) \geq\left(1-p_{i-1}^{*}\right) Q(x)$. Thus,

$$
P(x)>p_{i-1}(x)=\sum_{j=1}^{i-1} \alpha_{j}(x) \geq(i-1)\left(1-p_{i-1}^{*}\right) Q(x),
$$

which implies that

$$
i \leq \frac{1}{1-p_{i-1}^{*}} \cdot \frac{P(x)}{Q(x)}+1
$$

We have shown this inequality assuming $i \geq 2$ and $\alpha_{i}(x)>0$, but clearly it holds when $i=1$ and $\alpha_{1}(x)>0$. Returning to 
(IV.3) with this, we obtain

$$
\begin{aligned}
& \mathbb{E}[\lg i]=\sum_{x \in \mathcal{X}} \sum_{i=1}^{\infty} \alpha_{i}(x) \cdot \lg i \\
& \leq \sum_{x \in \mathcal{X}} \sum_{i=1}^{\infty} \alpha_{i}(x) \cdot \lg \left(\frac{1}{1-p_{i-1}^{*}} \cdot \frac{P(x)}{Q(x)}+1\right) \\
& \text { [by (IV.4)] } \\
& \leq \sum_{x \in \mathcal{X}} \sum_{i=1}^{\infty} \alpha_{i}(x) \cdot \lg \left(\frac{1}{1-p_{i-1}^{*}}\left(\frac{P(x)}{Q(x)}+1\right)\right) \\
& \text { [since } 0 \leq p_{i-1}^{*} \leq 1 \text { ] } \\
& =\sum_{i=1}^{\infty}\left(p_{i}^{*}-p_{i-1}^{*}\right) \cdot \lg \left(\frac{1}{1-p_{i-1}^{*}}\right)+ \\
& \sum_{x \in \mathcal{X}} P(x) \cdot \lg \left(\frac{P(x)}{Q(x)}+1\right) \\
& \leq \int_{0}^{1} \lg \frac{1}{1-p} \mathrm{~d} p+\sum_{x \in \mathcal{X}} P(x) \cdot \lg \left(\frac{P(x)}{Q(x)}+1\right) \\
& =\lg e+\sum_{x \in \mathcal{X}} P(x) \lg \left(\frac{P(x)}{Q(x)}\right)+ \\
& \sum_{x \in \mathcal{X}} P(x) \lg \left(1+\frac{Q(x)}{P(x)}\right) \\
& \leq \lg e+S(P \| Q)+\sum_{x \in \mathcal{X}} P(x) \cdot \lg \exp \left(\frac{Q(x)}{P(x)}\right) \\
& \text { [since } 1+t \leq \exp (t) \text { ] } \\
& =S(P \| Q)+2 \lg e \text {. }
\end{aligned}
$$

\section{Proof of Direct Sum Result (Result 3)}

Below we present our result in the two-party model for computing functions $f: \mathcal{X} \times \mathcal{Y} \rightarrow \mathcal{Z}$. However, the result also holds for protocols computing relations $R \subseteq \mathcal{X} \times \mathcal{Y} \times \mathcal{Z}$ in which Alice and Bob given $x \in \mathcal{X}$ and $y \in \mathcal{Y}$ respectively, need to output a $z \in \mathcal{Z}$ such that $(x, y, z) \in R$.

Our proof uses the notion of information cost defined by Chakrabarti et al. [CSWY01], and refined in several subsequent works [BJKS04], [JRS03a], [JRS03b], [JRS05].

Definition V.1 (Information cost). Let $\Pi$ be a private coins protocol taking inputs from the set $\mathcal{X} \times \mathcal{Y}$, and let $\mu$ be a distribution on the input set $\mathcal{X} \times \mathcal{Y}$. Then, the information cost of $\Pi$ under $\mu$ is

$$
\mathrm{IC}^{\mu}(\Pi)=I[X Y: M]
$$

where $(X, Y)$ represent the input to the two parties (chosen according to the distribution $\mu$ ) and $M$ is the transcript of the messages exchanged by the protocol on this input. For a function $f: \mathcal{X} \times \mathcal{Y} \rightarrow \mathcal{Z}$, let

$$
\mathrm{IC}_{\varepsilon}^{\mu, k}(f)=\min _{\Pi} \mathrm{IC} \mathrm{C}^{\mu}(\Pi),
$$

where $\Pi$ ranges over all $k$-round private-coins protocols for $f$ with error at most $\varepsilon$ under $\mu$.
We immediately have the following relationship between $\mathrm{IC}_{\varepsilon}^{\mu, k}$ and $D_{\varepsilon}^{\mu, k}$.

Proposition V.2. $I C_{\varepsilon}^{\mu, k}(f) \leq D_{\varepsilon}^{\mu, k}(f)$.

Proof: Let $\Pi$ be a protocol whose communication is $c \triangleq$ $D_{\varepsilon}^{\mu, k}(f)$. Let $M$ denote the message transcript of $\Pi$. Then we have, $c \geq H(M) \geq I[X Y: M] \geq \mathrm{IC}_{\varepsilon}^{\mu, k}(f)$.

A key insight of Chakrabarti et al. [CSWY01] was that one could show (approximately) a relationship in the opposite direction when the inputs are being drawn from the uniform distribution. They showed this for SMP protocols using a kind of message compression. Their result was then extended using different techniques involving the (classical) substate theorem (Theorem I.9) by Jain et al. [JRS03a], [JRS05]. Using this they showed that messages could be compressed to the amount of information they carry about the inputs, under all distributions for one-way and SMP protocols and under product distributions for two-way protocols. These message compression results then lead to corresponding direct sum results. Using Result 1 , we can considerably strengthen the result of Jain et al. [JRS03a] for two-way protocols as follows.

Lemma V.3 (Message compression). Let $\varepsilon, \delta>0$. Let $\mu$ be a distribution (not necessarily product) on the $\mathcal{X} \times \mathcal{Y}$ and $f: \mathcal{X} \times \mathcal{Y} \rightarrow \mathcal{Z}$. Then,

$$
D_{\varepsilon+\delta}^{\mu, k}(f) \leq \frac{1}{\delta}\left[2 \cdot \mathrm{IC}_{\varepsilon}^{\mu, k}(f)+O(k)\right] .
$$

Jain et al. [JRS03a] also obtain a similar message compression result however the dependence on $k$, the number of rounds, in their result is much worse as mentioned in the Introduction section.

The second ingredient in our proof of Result 3 is the direct sum property of information cost, originally observed by Chakrabarti et al. [CSWY01] for the uniform distribution.

Lemma V.4 (Direct sum for information cost). Let $\mu$ be a product distribution on $\mathcal{X} \times \mathcal{Y}$. Then, $\mathrm{IC}_{\varepsilon}^{\mu^{t}, k}\left(f^{(t)}\right) \geq t$. $\mathrm{IC}_{\varepsilon}^{\mu, k}(f)$.

This is the only place in the proof where we require $\mu$ to be a product distribution.

Before proving these lemmas, let us show that they immediately imply our theorem.

Proof of Result 3: Let $\mu$ be a product distribution on $\mathcal{X} \times \mathcal{Y}$. Then we have

$$
\begin{aligned}
D_{\varepsilon}^{\mu^{t}, k}\left(f^{(t)}\right) & \geq \mathrm{IC}_{\varepsilon}^{\mu^{t}, k}\left(f^{(t)}\right) \\
& \geq t \cdot \mathrm{IC}_{\varepsilon}^{\mu, k}(f) \\
& \geq \frac{t}{2}\left(\delta D_{\varepsilon+\delta}^{\mu, k}(f)-O(k)\right),
\end{aligned}
$$

where the first inequality follows from Proposition V.2, the second from Lemma V.4 and the last from Lemma V.3.

Proof of Lemma V.3: Let $\mu$ be a distribution on $\mathcal{X} \times$ $\mathcal{Y}$. Fix a private-coins protocol $\Pi$ that achieves the optimum information cost $\mathrm{IC}_{\varepsilon}^{\mu, k}(f)$. Let $(X, Y)$ be the random variables representing the inputs of Alice and Bob distributed according to $\mu$. We will use the following notation: $M=M(X, Y)$ will be the transcript of the protocol; for $i=1,2, \ldots, k, M_{i}$ will 
denote the $i$-th message of the transcript $M$ and $M_{1, i}$ will denote the first $i$ messages in $M$. Now, we have from the chain rule for mutual information (cf. [CT91]).

$$
I[X Y: M]=\sum_{i=1}^{k} I\left[X Y: M_{i} \mid M_{1, i-1}\right] .
$$

We now construct another protocol $\Pi^{\prime}$ as follows. The idea is as follows. For $i=1,2, \ldots, k$, the party that sent $M_{i}$ in $\Pi$, will now instead use Result 1 to generate the message $M_{i}$ for the other party by sending about $I\left[X Y: M_{i} \mid M_{1, i-1}\right]$ bits on the average. Suppose, we manage to generate the first $i-1$ messages in $\Pi^{\prime}$ with distribution exactly as that of $M_{1, i-1}$, and the (partial) transcript so far is $m$. For the rest of this paragraph we condition on $M_{1, i-1}=m$, and describe how the next message is generated. Assume that it is Alice's turn to send the next message. We have two observations concerning the distributions involved. First, the prefix $m$ of the transcript has already been generated and hence both parties can condition on this information. In particular, the conditional distribution $\left(M_{i} \mid M_{1, i-1}=m\right)$ is known to both Alice and Bob and (pregenerated) samples from it can be used as shared randomness. Second, since $\Pi$ is a private-coins protocol, for each $x \in \mathcal{X}$, the conditional random variable $\left(M_{i}(x, Y) \mid M_{1, i-1}(x, Y)=\right.$ $m)$, is independent of $Y$. Hence on input $x$, Alice knows the distribution of $\left(M_{i}(x, Y) \mid M_{1, i-1}(x, Y)=m\right)$.

The second observation in particular implies (using chain rule for information),

$$
I\left[X Y: M_{i} \mid M_{1, i-1}=m\right]=I\left[X: M_{i} \mid M_{1, i-1}=m\right] .
$$

Thus, by Result 1 , Alice can arrange for $\left(M_{i} \mid M_{1, i-1}=m\right)$ to be generated on Bob's side by sending at most

$$
2 I\left[X: M_{i} \mid M_{1, i-1}=m\right]+O(1)
$$

bits on the average; the overall communication in the $i$-th round is the average of this quantity over all choices $m$, that is, at most

$$
2 I\left[X Y: M_{i} \mid M_{1, i-1}\right]+O(1) .
$$

By applying this strategy for all rounds, we note from (V.1) that we obtain a public-coins $k$-round protocol $\Pi^{\prime}$, with expected communication $2 I[X Y: M]+O(k)$ bits, and error at most $\varepsilon$ as in $\Pi$. Using Markov's inequality, we conclude that the number of bits sent by the protocol is at least $\frac{1}{\delta}$ times this quantity with probability at most $\delta$. By truncating the long runs and then fixing the private random sequences suitably, we obtain a deterministic protocol $\Pi^{\prime \prime}$ with error at most $\varepsilon+\delta$ and communication at most $\frac{1}{\delta}(2 I[X Y: M]+O(k))=$ $\frac{1}{\delta}\left(2 \cdot I C_{\varepsilon}^{\mu, k}(f)+O(k)\right)$. The lemma now follows from this and the definition of $D_{\varepsilon+\delta}^{\mu, k}(f)$.

Proof of Lemma V.4: Let $\mu$ be a product distribution on $\mathcal{X} \times \mathcal{Y}$. Fix a $k$-round private-coins protocol $\Pi$ for $f^{(t)}$ that achieves $\mathrm{IC}_{\varepsilon}^{\mu^{t}, k}\left(f^{(t)}\right)$. For this protocol $\Pi$ the input is chosen according to $\mu^{t}$. We denote this input by $(X, Y)=\left(X_{1} X_{2} \cdots X_{t}, Y_{1} Y_{2} \cdots Y_{t}\right)$ and note that the $2 t$ random variables involved are mutually independent. Let $M$ denote the transcript of this protocol when run on the input
$(X, Y)$. Now, we have from chain rule for mutual information and independence of the $2 t$ random variables as above,

$$
\mathrm{IC}_{\varepsilon}^{\mu^{k}, k}(f)=I[X Y: M] \geq \sum_{i=1}^{t} I\left[X_{i} Y_{i}: M\right] .
$$

We claim that each term in the sum of the form $I\left[X_{i} Y_{i}: M\right]$ is at least $\mathrm{IC}_{\varepsilon}^{\mu, k}$. Indeed, consider the following protocol $\Pi^{\prime}$ for $f$ derived from $\Pi$. In $\Pi^{\prime}$, on receiving the input $(x, y) \in$ $\mathcal{X} \times \mathcal{Y}$, Alice and Bob simulate $\Pi$ as follows. They insert $x$ and $y$ as the $i$-th component of their respective inputs for $\Pi$, and generate the remaining components based on the product distribution $\mu$. They can do so using private coins since $\mu$ is a product distribution. This results in a $k$-round private coins protocol $\Pi^{\prime}$ for $f$ with error at most $\varepsilon$ under $\mu$, since the error of $\Pi$ was at most $\varepsilon$ under $\mu^{k}$. Clearly, $\mathrm{IC}^{\mu}(\Pi)=I\left[X_{i} Y_{i}: M\right]$.

\section{Separating $T(X: Y), C(X: Y)$ And $I[X: Y]$}

For any pair of random variables $(X, Y)$, it easily follows from the definitions that $T(X: Y) \geq C(X: Y)$. Furthermore, by Wyner's theorem (Theorem I.1)

$$
C(X: Y)=\min _{W} I[X Y: W],
$$

where $W$ is such that $X$ and $Y$ are independent when conditioned on $W$. Note, however, that

$$
I[X Y: W] \geq I[X: W] \geq I[X: Y] .
$$

The first inequality comes from the monotonicity of mutual information which in turn follows from the chain rule for mutual information. The second inequality is the data processing inequality applied to the Markov chain $X \rightarrow W \rightarrow Y$. Thus, we have $T(X: Y) \geq C(X: Y) \geq I[X: Y]$. In this section, we will show that both these inequalities are strict for $(X, Y)$ defined as follows.

Definition VI.1. Let $W=(i, b)$ be a random variable uniformly distributed over the set $[n] \times\{0,1\}$. Now, let $X$ and $Y$ be random variables taking values in $\{0,1\}^{n}$, such that

(a) $\operatorname{Pr}[X=z \mid W=(i, b)], \operatorname{Pr}[Y=z \mid W=(i, b)]$ $\begin{cases}2^{-(n-1)} & z[i]=b \\ 0 & \text { otherwise }\end{cases}$

(b) $X$ and $Y$ are independent when conditioned on $W$.

It is easy to see that $X$ and $Y$ are uniformly distributed $n$-bit strings (but not independent). Hence, $H[X]=H[Y]=n$.

Proposition VI.2. For $(X, Y)$ defined as above, we have:

(a) $I[X: Y]=O\left(n^{-\frac{1}{3}}\right)$.

(b) $C(X: Y)=2-I[X: Y]=2-O\left(n^{-\frac{1}{3}}\right)$.

(c) $T(X: Y)=\Theta(\lg n)$.

Note that in the above example, though $C(X: Y)$ and $I[X$ : $Y$ ] differ by a super-constant multiplicative factor, they only differ by a constant additive term. We can construct another joint distribution $\left(X^{\prime}, Y^{\prime}\right)$ by taking $m$ independent copies of the joint distribution $(X, Y)$ (i.e., $\left.\left(X^{\prime}, Y^{\prime}\right)=(X, Y)^{m}\right)$. It then follows from the chain rule for mutual information that 
$I\left[X^{\prime}: Y^{\prime}\right]=I\left[X^{m}: Y^{m}\right]=m I[X: Y]=o(m)$. However, $C\left(X^{\prime}: Y^{\prime}\right)=C\left(X^{m}: Y^{m}\right)=m C(X: Y)=\Theta(m)^{4}$. This implies that it is not possible to bound $C(X: Y)$ from above by a linear function in $I[X: Y]$.

Proof of part (a): Given $X=x$ for some $n$-bit string $x$, the conditional distribution $\left.Y\right|_{X=x}$ is given by

$$
\operatorname{Pr}[Y=y \mid X=x]=\frac{\operatorname{agr}(x, y)}{n 2^{n-1}}
$$

where $\operatorname{agr}(x, y)$ is the number of bit positions $x$ and $y$ agree on. We can now compute the conditional entropy $H[Y \mid X]$ as follows:

$$
\begin{aligned}
& H[Y \mid X]=-\sum_{x \in\{0,1\}^{n}} \frac{1}{2^{n}} \sum_{k=0}^{n}\left(\begin{array}{l}
n \\
k
\end{array}\right) \frac{k}{n 2^{n-1}} \lg \frac{k}{n 2^{n-1}} \\
& =-\sum_{k=0}^{n}\left(\begin{array}{l}
n \\
k
\end{array}\right) \frac{k}{n 2^{n-1}} \lg \frac{k}{n 2^{n-1}} \\
& =-\sum_{k=1}^{n}\left(\begin{array}{l}
n-1 \\
k-1
\end{array}\right) \frac{1}{2^{n-1}} \lg \frac{k}{n 2^{n-1}} \\
& =-\sum_{k=0}^{n-1}\left(\begin{array}{c}
n-1 \\
k
\end{array}\right) \frac{1}{2^{n-1}} \text {. } \\
& {[\lg (k+1)-(n+\lg n-1)]} \\
& =n+\lg n-1-\sum_{k=0}^{n-1}\left(\begin{array}{c}
n-1 \\
k
\end{array}\right) \frac{1}{2^{n-1}} \lg (k+1) \\
& \geq n+\lg n-1-\left(1-2^{-O\left(n^{1 / 3}\right)}\right) \text {. } \\
& \lg \left[\frac{n}{2}\left(1+\frac{1}{n^{1 / 3}}\right)\right]-2^{-O\left(n^{1 / 3}\right)} \cdot \lg n \\
& =n+\lg n-1 \\
& -\left(1-2^{-O\left(n^{1 / 3}\right)}\right) \cdot\left(\lg n-1+\frac{\lg e}{n^{1 / 3}}\right) \\
& -2^{-O\left(n^{1 / 3}\right)} \cdot \lg n \\
& \text { [ since } \lg (1+\delta) \leq \delta \lg e] \\
& =n-O\left(\frac{1}{n^{1 / 3}}\right)
\end{aligned}
$$

Thus, $I[X: Y]=H[Y]-H[Y \mid X]=O\left(n^{-\frac{1}{3}}\right)$.

Proof of part (b): By Wyner's theorem (Theorem I.1),

$$
\begin{aligned}
C(X: Y) & =\min _{W^{\prime}} I\left[X Y: W^{\prime}\right] \\
& =H[X Y]-\max _{W^{\prime}} H\left[X Y \mid W^{\prime}\right] \\
& =H[X]+H[Y]-I[X: Y]-\max _{W^{\prime}} H[X Y \mid \\
& =2 n-I[X: Y]-\max _{W^{\prime}} H\left[X Y \mid W^{\prime}\right] .
\end{aligned}
$$

where the random variable $W^{\prime}$ is such that $I\left[X: Y \mid W^{\prime}\right]=0$. We already know that $I[X: Y]=O\left(n^{-\frac{1}{3}}\right)$. So, part (b) will follow if we show

$$
\max _{W^{\prime}} H\left[X Y \mid W^{\prime}\right]=2 n-2 .
$$

${ }^{4} C\left(X^{m}: Y^{m}\right)=\liminf \operatorname{in}_{\lambda \rightarrow 0} \lim _{k \rightarrow \infty}\left(T\left(X^{m k}: Y^{m k}\right) / k\right)=m$. $\liminf _{\lambda \rightarrow 0} \lim _{k \rightarrow \infty}\left(T\left(X^{m k}: Y^{m k}\right) / m k\right)=m C(X: Y)$ where the first and third equalities follow from (I.1)
Let $W^{\prime}$ be such that $I\left[X: Y \mid W^{\prime}\right]=0$. Consider any $w$ in the support of $W^{\prime}$. Let $X_{w}$ be the set of $x \in\{0,1\}^{n}$ such that $\operatorname{Pr}\left[X=x \mid W^{\prime}=w\right]>0$. Similarly, define $Y_{w}$. We must have that $\left|X_{w}\right|+\left|Y_{w}\right| \leq 2^{n}$, since otherwise there must exist an $x$ such that $\operatorname{Pr}[X=x \wedge Y=\bar{x}]>0$ where $\bar{x}$ is the $n$-bit string obtained by complementing each bit of $x$. This implies that $\left|X_{w} \times Y_{w}\right| \leq 2^{2 n} / 4$. Thus,

$$
\max _{W^{\prime}} H\left[X Y \mid W^{\prime}\right] \leq 2 n-2 .
$$

Now, if we let $W^{\prime}$ be the random variable $W$ used in Definition VI.1, we have $H[X Y \mid W]=2(n-1)$. Hence,

$$
\max _{W^{\prime}} H\left[X Y \mid W^{\prime}\right] \geq 2 n-2 .
$$

This justifies (VI.1) and completes the proof of part (b).

To prove part (c), we will use a theorem of Harper [Har66], which states that Hamming balls in the hypercube have the smallest boundary. The following version, due to Frankl and Füredi (see Bollobás [Bol86, Theorem 3, page 127]), will be the most convenient for us. First, we need some notation.

e) Notation:: For $x, y \in\{0,1\}^{n}$, let $d(x, y)$ be the Hamming distance between $x$ and $y$, that is, the number of positions where $x$ and $y$ differ. For non-empty subsets $\mathcal{A}, \mathcal{B} \subseteq\{0,1\}^{n}$, let

$$
d(\mathcal{A}, \mathcal{B}) \triangleq \min \{d(a, b): a \in \mathcal{A} \text { and } b \in \mathcal{B}\} .
$$

We say that a subset $S \subseteq\{0,1\}^{n}$ is a Hamming ball centered at $x \in\{0,1\}^{n}$ if for all $y, y^{\prime} \in\{0,1\}^{n}$, if $y \in S$ and $d\left(x, y^{\prime}\right)<d(x, y)$, then $y^{\prime} \in S$. Let

$$
\operatorname{Ball}(x, d)=\left\{y \in\{0,1\}^{n}: d(x, y) \leq d\right\} .
$$

Theorem VI.3 ([Bol86, Theorem 3, page 127]). Let $\mathcal{A}$ and $\mathcal{B}$ be non-empty subsets of $\{0,1\}^{n}$. Then, we can find Hamming balls $\mathcal{A}_{0}$ and $\mathcal{B}_{0}$ centered at $0^{n}$ and $1^{n}$ respectively, such that $\left|\mathcal{A}_{0}\right|=|\mathcal{A}|,\left|\mathcal{B}_{0}\right|=|\mathcal{B}|$, and $d\left(\mathcal{A}_{0}, \mathcal{B}_{0}\right) \geq d(\mathcal{A}, \mathcal{B})$.

Corollary VI.4. If $\mathcal{A}$ and $\mathcal{B}$ are non-empty sets of strings such that $d(\mathcal{A}, \mathcal{B}) \geq d \geq 2$, then

$$
\min \{|\mathcal{A}|,|\mathcal{B}|\} \leq \exp \left(-\frac{(d-2)^{2}}{2 n}\right) 2^{n} .
$$

Proof: By Theorem VI.3, we may assume that $\mathcal{A}$ and $\mathcal{B}$ are balls centered at $0^{n}$ and $1^{n}$. Suppose $|\mathcal{A}| \leq|\mathcal{B}|$, and let $r$ be a non-negative integer such that

$$
\operatorname{Ball}\left(0^{n}, r\right) \subseteq \mathcal{A} \subseteq \operatorname{Ball}\left(0^{n}, r+1\right) .
$$

Then, $2 r+d \leq n$, that is, $r+1 \leq(n-d+2) / 2$. It then follows $W^{\prime}$ ] using the Chernoff bound (see, e.g., Alon and Spencer [AS00, Theorem A.1.1, page 263]) that

$$
|\mathcal{A}| \leq\left|\operatorname{Ball}\left(0^{n}, r+1\right)\right| \leq \exp \left(-\frac{(d-2)^{2}}{2 n}\right) \cdot 2^{n} .
$$

Proof of part (c): It is easy to see that $T(X: Y) \leq$ $\lceil\lg n\rceil+1$ : on receiving $x \in\{0,1\}^{n}$, Alice sends Bob an index $i$ uniformly distributed in $[n]$ and the bit $x[i]$; on receiving $(i, b)$, Bob generates a random string $y \in\{0,1\}$ such that $y[i]=b$, with each of the $2^{n-1}$ possibilities being equally likely. 
It remains to show that $T(X: Y)=\Omega(\lg n)$. It follows from the definition of $T(X: Y)$ that $T(X: Y) \geq \min _{W^{\prime}} H\left[W^{\prime}\right]$, where the minimum is over all random variables $W^{\prime}$ such that $X$ and $Y$ are conditionally independent given $W^{\prime}$. Thus, it is enough to show that any such $W^{\prime}$ has entropy $\Omega(\lg n)$. Let $W^{\prime}$ be one such random variable. We show below that for all $w$

$$
\operatorname{Pr}\left[W^{\prime}=w\right]=O\left(\sqrt{\frac{\lg n}{n}}\right) .
$$

That is, we show that the min-entropy of $W^{\prime}$ is $\Omega(\lg n)$; it follows that the entropy of $W^{\prime}$ is $\Omega(\lg n)$

Fix $w$ such that $\alpha \triangleq \operatorname{Pr}\left[W^{\prime}=w\right]>0$. Let

$$
\begin{aligned}
& X_{w}=\left\{x \in\{0,1\}^{n}: \operatorname{Pr}\left[X=x \mid W^{\prime}=w\right]>2^{-(n+1)}\right\} ; \\
& Y_{w}=\left\{y \in\{0,1\}^{n}: \operatorname{Pr}\left[X=y \mid W^{\prime}=w\right]>2^{-(n+1)}\right\} .
\end{aligned}
$$

Then, for all $x \in X_{w}$ and $y \in Y_{w}$, we have

$$
\begin{aligned}
\alpha 2^{-2(n+1)} & <\operatorname{Pr}\left[(X, Y)=(x, y) \wedge W^{\prime}=w\right] \\
& \leq \operatorname{Pr}[(X, Y)=(x, y)] \\
& =\frac{\operatorname{agr}(x, y)}{n 2^{2 n-1}},
\end{aligned}
$$

that is, $\operatorname{agr}(x, y)>\alpha n / 8$. Furthermore, since for all $x$, $\operatorname{Pr}\left[X=x \mid W^{\prime}=w\right] \leq 2^{-n} / \alpha$ and $\sum_{x \in\{0,1\}^{n}} \operatorname{Pr}[X=$ $\left.x \mid W^{\prime}=w\right]=1$, we have $\left|X_{w}\right| \geq \alpha 2^{n-1}$. Similarly $\left|Y_{w}\right| \geq \alpha 2^{n-1}$. We thus obtain two sets $X_{w}, Y_{w} \subseteq\{0,1\}^{n}$, each with at least $\alpha 2^{n-1}$ elements, such that every $x \in X_{w}$ and $y \in Y_{w}$ satisfies $\operatorname{agr}(x, y)>\alpha n / 8$. Our goal is to show that this implies that $\alpha$ is small.

Let $Y_{w}^{\prime}$ be the set of strings whose complements belong to $Y_{w}$. Since $\operatorname{agr}(x, y)>\alpha n / 8$ for all $x \in X_{w}$ and $y \in Y_{w}$, the Hamming distance between $X_{w}$ and $Y_{w}^{\prime}$ is more than $\alpha n / 8$. By Corollary VI.4, we conclude that

$$
\alpha 2^{n-1} \leq \exp \left(-\frac{(\alpha n-16)^{2}}{128}\right) 2^{n}
$$

which implies that $\alpha=O\left(\sqrt{\frac{\ln n}{n}}\right)$, for all large enough $n$.

\section{REDUCING THE SHARED RANDOMNESS}

In the preceding sections, we did not formally bound the amount of shared randomness used by the protocol. We now address this shortcoming, and show how one can reduce the number of shared random bits used substantially, while increasing the communication only slightly. Our main result is the following.

Theorem VII.1. For all pairs of random variables $(X, Y)$, there is a one-way protocol $\Pi$ for generating $(X, Y)$ (so that $(X, \Pi(X))$ has the same distribution as $(X, Y))$ such that

1) the expected communication from Alice to Bob is at most $I[X: Y]+O(\lg (I[X \quad: \quad Y]+1))+$ $O(\lg \lg |\operatorname{support}(Y)|)$;

2) the number of bits of shared randomness read by either party is $O(\lg \lg |\operatorname{support}(X)|+\lg |\operatorname{support}(Y)|)$.
To justify this theorem, we will present a protocol that satisfies the requirements. We will derive our semiprotocol from a probabilistic argument, which we state using the language of graphs.

Definition VII.2 (Protocol graphs). A protocol graph $G(M, N)$ is a labeled directed acyclic graph with a source $s$, a sink $t$, and two layers in between: $V$ with $M$ vertices and $W$ with $N$. The source $s$ is connected by an edge to each of the $M$ vertices in the layer $V$. Each of the $N$ vertices in the third layer $W$ is connected to the sink $t$. The remaining edges go from $V$ to $W$. We use $E$ to denote the set of these edges. There is a labeling $\ell: E \rightarrow\{0,1\}^{*}$, such that for each $v \in V$, the labeling $\ell$ when restricted to the edges incident on $v$ is a prefix-free encoding for those edges.

In our argument, Alice and Bob will work based on a graph $G(M, N)$, viewing $[M]$ as the set of shared random strings, and $[N]$ as the set over which Bob's output must be distributed. The edges of the graphs and the labels on them will determine how Bob interprets Alice's message. This is made precise in the following definition.

Definition VII.3 (Protocols based on graphs). Let $G(M, N)$ be a protocol graph and let $P$ be a distribution on $[N]$. In a protocol for $P$ based on $G$. Alice sends a message to Bob so as to enable him to generates a string $w \in[N]$ whose distribution is $P$. Such a protocol operates as follows.

- Shared randomness: Alice and Bob share a random string $R$ picked with uniform distribution from $[M]$, so $R$ has $\lceil\lg M\rceil$ bits;

- Message: Alice computes her message $m \in\{0,1\}^{*}$ based on the random string, her input and her private coins;

- Output: On receiving the message $m \in\{0,1\}^{*}$, Bob outputs $w \in[N]$, where $(R, w)$ is the unique edge of $G$ incident on $R$ with label $m$.

The cost of a protocol is the expected number of bits Alice transmits.

Lemma VII.4 (Main lemma). For all distributions $Q$ on $[N]$ and $M \geq N$, there is a distribution $G(M, N)$ on protocol graphs, such that for each distribution $P$ on $[N]$, with probability greater than $1-2^{-M}$, there is a protocol for $P$ based on $G$ with cost $S(P \| Q)+O(\lg (S(P \| Q)+1))+O(\lg \lg N)$.

Before proving this lemma, let us first see how this immediately implies Theorem VII.1.

Proof of Theorem VII.1: We apply Lemma VII.4 with $Q$ as the distribution of $Y, P$ as $P_{x}$, the distribution of $Y$ conditioned on $X=x$, and $M=$ $\max \{\lceil\lg |\operatorname{support}(X)|\rceil,|\operatorname{support}(Y)|\}$. Since there are at most $2^{M}$ choices for $x \in \operatorname{support}(X)$, we conclude from the union bound that there is an instance $\hat{G}$ of the protocol graph $G(M, N)$, such that for each $x \in \operatorname{support}(X)$, there is a protocol $\Pi_{x}$ for $P_{x}$ based on $\hat{G}$, using $O(\lg M)$ bits of shared randomness and $S\left(P_{x} \| Q\right)+O\left(\lg \left(S\left(P_{x} \| Q\right)+1\right)\right)+O(\lg \lg N)$ bits of communication. Since Bob's actions are determined completely by the protocol graph, he acts in the same way in all these protocols. The protocol for $(X, Y)$ is now straight- 
forward: on input $x$, Alice sends a message assuming she is executing $\Pi_{x}$, and Bob interprets this message as before using the graph $\hat{G}$, and is guaranteed to output a string $y \in$ support $(Y)$ with distribution $P_{x}$. We thus have a protocol for $(X, Y)$. Furthermore, it follows from Fact II.1 (see Section II) that the cost of this protocol is $I[X: Y]+O(\lg I[X$ : $Y])+O(\lg \lg N)$. The number of bits of shared randomness is $\lceil\lg M\rceil=O(\lg \lg |\operatorname{support}(X)|+\lg |\operatorname{support}(Y)|)$.

\section{A. Proof of Lemma VII.4}

To prove Lemma VII.4, we will view a protocol with low communication as a low-cost flow in a suitably constructed capacitated protocol graph. Then, we will construct a random graph that admits such a low-cost flow with high probability.

Definition VII.5 (Capacities, flows). Let $G(M, N)$ be a protocol graph and $P$ a distribution on $[N]$. Then, $G^{P}$ is the capacitated version of $G$ where the edges of the form $(s, v)$ have capacity $\frac{1}{M}$, edges of the form $(w, t)$ have capacity $P(w)$, and all other edges have infinite capacity. We represent these capacities by the map $\ell: E \rightarrow \mathbb{R}$. An $\alpha$-flow for the distribution $P$ based on the graph $G(M, N)$ is a flow $f: E(G) \rightarrow[0,1]$ in $G^{P}$ such that the total flow is at least $\alpha$. We write just flow instead of 1-flow. The cost of a flow $f$ is $\sum_{e \in E} f(e)|\ell(e)|$.

Proposition VII.6. Let $G(M, N)$ be a protocol graph and $P$ be a distribution on $[N]$. If there is a flow in $G$ for $P$ with cost $C$, then there is a protocol for $P$ with shared randomness $O(\lg M)$ and cost $C$.

Proof: Fix a flow $f$ in $G$ for $P$. We will show how Alice picks the label to transmit in order to enable Bob to generate a string in $[N]$ with the required distribution. If the shared random string is $R$, Alice picks the edge $e=(R, w)$ leaving $R$ with probability $M f(e)$ and transmits its label $\ell(e)$. Bob's actions are now determined, and it is easy to verify that the string he produces has the required distribution.

Now, Lemma VII.4 follows immediately by combining the above proposition with the following lemma, which is the main technical observation of this section.

Lemma VII.7. For all distributions $Q$ on $[N]$ and $M \geq N$, there is a random variable $\mathbf{G}(M, N)$ each of whose instances is a protocol graph $G(M, N)$, such that for each distribution $P$ on $[N]$, with probability at least $1-2^{-M}$, there is a flow for $P$ based on $\mathbf{G}$ with cost $S(P \| Q)+O(\lg S(P \| Q))+$ $O(\lg \lg N)$.

Proof: To define the random graph $G(M, N)$, we need to define the set, $E(V, W)$ of edges that go from $V=[M]$ to $W=[N]$, and their labeling. In our random graph, $E(V, W)$ will be the union of two sets $E_{0}$ and $E_{1}$; the labels of the edges in $E_{0}$ begin with a 0 and the labels of the edges in $E_{1}$ begin with a 1 .

- The edges in $E_{0}: E_{0}=[M] \times[N]$, with the edge $(i, j)$ labeled by $0 \cdot[j]$, where $[j]$ denotes the binary encoding of $j$ using $\lceil\lg N\rceil$ bits;

- The edges in $E_{1}$ : The labels of the edges in this set start with a 1 . These edges are generated randomly as follows.
For each $i \in[M]$ and each $k \in \mathbb{Z}^{+}$, we have one edge with label $1 \cdot \tau(k)$, where $\tau: \mathbb{Z}^{+} \rightarrow\{0,1\}^{*}$ is a prefix-free encoding of $\mathbb{Z}^{+}$with $|\tau(k)| \leq \lg k+O(\lg (1+\lg k))$. The other end point of this edge is chosen randomly from the set $[N]$ with distribution $Q$ (independently for different $i$ and $k$ ).

We wish to show that for all distributions $P$ on $[N]$, with high probability, there is a low-cost flow in $G$ for $P$ (that is, a flow of value 1 in $G^{P}$ ). We will do this in two steps. First, we will show using the max-flow min-cut theorem that with high probability there is a low-cost $\left(1-\frac{1}{\lg N}\right)$-flow in $G$ for $P$ using the edges in $E_{1}$. To turn this into a proper flow, we will send some additional flow along the edges in $E_{0}$. Since the total value of the flow along edges in $E_{0}$ is $\left(\frac{1}{\lg N}\right)$, this will not significantly increase the cost.

Consider the subgraph $G_{1}$ of $G^{P}(M, N)$ obtained by retaining only those edges $(i, j)$ in $E_{1}$ whose labels lie in the set $\{1 \cdot \tau(1), 1 \cdot \tau(2), \ldots, 1 \cdot \tau(L(j))\}$, where $L(j) \triangleq$ $\left\lceil 3(P(j) / Q(j)) \lg ^{2} N\right\rceil$. To show that $G_{1}$ has a $\left(1-\frac{1}{\lg N}\right)$ flow with high probability, we show that (with high probability) it has no cut of size $1-\frac{1}{\lg N}$, that is the removal of no set of edges of total capacity less than $1-\frac{1}{\lg N}$ can disconnect $t$ from $s$. Since the edges going between $[M]$ and $[N]$ have infinite capacity, each edge in any such cut is incident on either $s$ or $t$. Fix a set of edges $\mathcal{C}$ of total capacity less than $1-\frac{1}{\lg N}$. Let $S=\{i \in V:(s, i) \notin \mathcal{C}\}$ and $T=\{j \in W:(j, t) \notin \mathcal{C}\}$. We will show that with high probability $\mathcal{C}$ is not an $s$ - $t$ cut in $G_{1}$. Note that $|S|>\frac{M}{\lg N}$ and $\sum_{i \in T} P(i)>\frac{1}{\lg N}$. For $\mathcal{C}$ to be a cut, there should be no edge in $G_{1}$ connecting $S$ and $T$, which happens with probability at most (recall that the edges in $E_{1}$ are generated independently):

$$
\begin{aligned}
\operatorname{Pr}\left[\mathcal{C} \text { is a cut in } G_{1}\right] & \leq \prod_{j \in T}(1-Q(j))^{L(j)|S|} \\
& \leq \prod_{j \in T} \exp (-L(j) Q(j)|S|) \\
& <\exp \left(-3\left(\lg ^{2} N\right)|S| \sum_{j \in T} P(j)\right) \\
& \leq \exp (-3 M) .
\end{aligned}
$$

Since there are at most $2^{M}$ choices for $S$ and at most $2^{N}$ choices for $T$, there are at most $2^{M+N}$ choices for $\mathcal{C}$. Thus, we have $\operatorname{Pr}\left[\right.$ there is a small cut in $\left.G_{1}^{P}\right]<2^{M+N} \exp (-3 M) \leq$ $2^{-M}$ (since $M \geq N$ ). By the max-flow min-cut theorem, with probability at least $1-2^{-M}, G_{1}$ has a flow with value at least $1-\frac{1}{\lg N}$ and cost $\sum_{j \in T} P(j)|\tau(L(j))|=S(P \| Q)+$ $O(\lg (S(P \| Q)+1))+O(\lg \lg N)$ (due to concavity of the $\lg (\cdot)$ function). We convert this $\left(\frac{1}{\lg N}\right)$-flow into a proper flow by using the edges in $E_{0}$ to supply the remaining $\frac{1}{\lg N}$ units. Since the edges in $E_{0}$ have labels of length at most $1+\lceil\lg N\rceil$ and the total flow through these edges is at most $\frac{1}{\lg N}$, the resulting increase in cost is $O(1)$. 


\section{ACKNOWLEDGEMENTS}

Prahladh Harsha, David McAllester and Jaikumar Radhakrishnan thank the members of the Winter 2006 machine learning reading group at TTI-Chicago for discussions that lead to this work. We thank Andreas Winter for informing us of his work with Charles Bennett on the asymptotic versions of our results, and Oded Regev for comments and suggestions that greatly helped in improving the presentation in this paper.

\section{REFERENCES}

[AS00] NOGA ALON and JOEL H SPENCER. The Probabilistic Method. Wiley-Interscience, second edition, 2000. doi:10.1002/0471722154.

[BJKS04] ZIV BAR-YosseF, T. S. JAYRAM, RAVI KUMAR, and D. SIVAKUMAR. An information statistics approach to data stream and communication complexity. Journal of Computer and System Sciences, 68(4):702-732, June 2004. (Preliminary Version in 43rd FOCS, 2002). doi:10.1016/j.jcss.2003.11.006.

[Bol86] BÉla Bollobás. Combinatorics: Set Systems, Hypergraphs, Families of Vectors and Combinatorial Probability. Cambridge University Press, 1986. doi:10.2277/0521337038.

[BSST02] Charles H. Bennett, Peter W. Shor, John A. Smolin, and Ashish V. THAPLIYAL. Entanglement-assisted capacity of a quantum channel and the reverse Shannon theorem. IEEE Transactions on Information Theory, 48(10):2637-2655, October 2002. (Preliminary Version in Proc. Quantum Information: Theory, Experiment and Perspectives Gdansk, Poland, 10 - 18 July 2001). doi:10.1109/TIT.2002.802612.

[BW06] Charles H. Bennett and Andreas Winter. Personal communication, 2006.

[CR04] Amit Chakrabarti and Oded Regev. An optimal randomised cell probe lower bound for approximate nearest neighbour searching. In Proceedings of the 45th IEEE Symposium on Foundations of Computer Science (FOCS), pages 473-482. Rome, Italy, 17-19 October 2004. doi:10.1109/FOCS.2004.12.

[CSWY01] Amit ChaKRABARTi, YaOYUn Shi, Anthony Wirth, and ANDREW CHI-CHIH YAO. Informational complexity and the direct sum problem for simultaneous message complexity. In Proceedings of the 42nd IEEE Symposium on Foundations of Computer Science (FOCS), pages 270-278. Las Vegas, Nevada, 14-17 October 2001. doi:10.1109/SFCS.2001.959901.

[CT91] Thomas M. COVER and Joy A. Thomas. Elements of Information Theory. Wiley-Interscience, 1991. doi:10.1002/0471200611.

[Har66] LAWRENCE H. HARPER. Optimal numberings and isoperimetric problems on graphs. J. Combinatorial Theory, 1(3):385-394, 1966. doi:10.1016/S0021-9800(66)80059-5.

[HJMR07] PRAHLADH HARSha, RAHUl JAIN, DAVID MCAllester, and JAIKUMAR RADHAKRISHNAN. The communication complexity of correlation. In Proceedings of the 22nd IEEE Conference on Computational Complexity, pages 10-23. San Diego, California, 13-16 June 2007. doi:10.1109/CCC.2007.32.

[Jai06] RAHUL JAIN. Communication complexity of remote state preparation with entanglement. Quantum Information and Computation, 6(4-5):461-464, July 2006. Available from: http://www. rintonpress.com/journals/qiconline.html.

[JRS02] RAHUL JAIN, JAIKUMAR RADHAKRISHNAN, and PRANAB SEN. Privacy and interaction in quantum communication complexity and a theorem about the relative entropy of quantum states. In Proceedings of the 43rd IEEE Symposium on Foundations of Computer Science (FOCS), pages 429-438. Vancouver, Canada, 16-19 November 2002. doi:10.1109/SFCS.2002.1181967.

[JRS03a] - A direct sum theorem in communication complexity via message compression. In JOS C. M. BAETEN, JAN KAREL Lenstra, JOACHIM PARrow, and Gerhard J. WOEGINGER, eds., Proceedings of the 30th International Colloquium of Automata, Languages and Programming (ICALP), volume 2719 of Lecture Notes in Computer Science, pages 300-315. Springer-Verlag, Eindhoven, Netherlands, 30 June-4 July 2003. doi:10.1007/3-540-45061-0_26.
[JRSO3b] A lower bound for the bounded round quantum communication complexity of set disjointness. In Proceedings of the 44th IEEE Symposium on Foundations of Computer Science (FOCS), pages 220-229. Cambridge, Massachusetts, 11-14 October 2003. doi:10.1109/SFCS.2003.1238196.

[JRS05] - Prior entanglement, message compression and privacy in quantum communication. In Proceedings of the 20th IEEE Conference on Computational Complexity, pages 285-296. San Jose, California, 12-15 June 2005. doi:10.1109/CCC.2005.24.

[KN97] Eyal Kushilevitz and NoAm Nisan. Communication Complexity. Cambridge University Press, 1997. doi: $10.2277 / 052102983 X$.

[Win02] ANDREAS WINTER. Compression of sources of probability distributions and density operators, 2002. arXiv:quant-ph/0208131.

[Wyn75] AARON D WYNER. The common information of two dependent random variables. IEEE Transactions on Information Theory, 21(2):163-179, March 1975.

[Yao77] ANDREW CHI-CHIH YAO. Probabilistic computations: Toward a unified measure of complexity (extended abstract). In Proceedings of the 18th IEEE Symposium on Foundations of Computer Science (FOCS), pages 222-227. Providence, Rhode Island, 31 October-1 November 1977. 